\title{
Neuropsychological Differences Related to Age in Dementia with Lewy Bodies
}

\author{
Yasuhiro Nagahama ${ }^{a}$ b Tomoko Okinab $^{b}$ Norio Suzuki ${ }^{b}$ \\ ${ }^{a}$ Kawasaki Memorial Hospital, Kawasaki City, Japan; ${ }^{b}$ Department of Geriatric Neurology, \\ Shiga Medical Center, Moriyama City, Japan
}

\section{Keywords}

Dementia with Lewy bodies · Memory · Executive function - Visuospatial abilities · Aging ·

Alzheimer disease

\section{Abstract}

Background/Aims: To examine the influence of age on neuropsychological performances in dementia with Lewy bodies (DLB) and Alzheimer disease (AD) patients. Methods: We examined memory, executive, and visuo-constructional performances in 202 DLB patients and 236 $A D$ patients. We divided the subjects into three age groups $(65-74,75-84$, and $85-95$ years old), and evaluated the differences in neuropsychological performances. Results: Recent memory in the DLB group was significantly better than that in the age-matched AD group when comparing the age groups 65-74 years and 75-84 years; however, memory impairment in the DLB patients in the age group 85-95 years was comparable with that in the agematched $A D$ patients. In contrast to recent memory, the other assessed neuropsychological performances, such as visuospatial and executive functions, showed no significant change in differences between the DLB and AD groups with advancing age. Conclusion: Our study revealed that the nature of memory impairment in DLB patients changes according to age. DLB patients in the young-old and old-old age groups showed significantly better memory performance than the age-matched AD patients, whereas memory performance of the DLB patients in the oldest-old age group was similar to that of the age-matched AD patients. This may be associated with the increased rate of coexisting AD pathology in DLB patients with older age. 
Nagahama et al.: Effect of Aging on Cognition in DLB Patients

\section{Introduction}

Dementia with Lewy bodies (DLB) is the second most common neurodegenerative dementia following Alzheimer disease (AD). In addition to memory disturbance, DLB is characterized by fluctuating cognition and attention, visual hallucinations, and parkinsonism [1]. A growing body of evidence indicates that patients with DLB show better memory performance and prominent attentional and visuospatial dysfunction compared with AD patients [2-4]. Careful neuropsychological assessment with clinical workup is becoming the fundamental standard for diagnosing DLB, and the "double dissociations" with relatively preserved memory performance and greater impairment of executive, visual perception, and performance tasks are clues to differentiate DLB from AD [1].

Autopsy study series revealed that the pathology of DLB is often concomitant with the pathology of AD. Mixed DLB/AD pathology has been shown to have a high prevalence [5]; this causes difficulties in clinical diagnosis, particularly in DLB patients with a high Braak stage who often lack core features of DLB, such as visual hallucinations [6].

In $\mathrm{AD}$, different phenotypic presentations have been noted according to the age of onset. Patients with early-onset AD (before the age of 65) tend to show greater impairments in attention, language, visuospatial, and executive functions, while patients with late-onset AD (after the age of 65) tend to show prominent deficits in episodic memory [7]. In contrast to $\mathrm{AD}$, there have been no studies examining the neuropsychological differences according to age in DLB patients.

The aims of the present study were to examine the differences in neuropsychological performance of DLB patients with different ages. We also examined AD patients at different ages for comparison with the DLB patients.

\section{Methods}

\section{Subjects}

We examined outpatients who attended the Memory Clinic, Shiga Medical Center, between July 2000 and July 2014. All subjects underwent general physical, neurological, and routine neuropsychological examinations; they also underwent structural neuroimaging (head CT or MRI) and routine laboratory investigations, including assessment of vitamin $\mathrm{B}_{1}, \mathrm{~B}_{12}$, and thyroid functions. Patients with a history of stroke, significant head trauma, alcohol abuse, major psychiatric illnesses, or evidence of other neurological disorders were excluded.

Patients with DLB were included consecutively, but patients with a Mini-Mental State Examination (MMSE) score of $<10$ were excluded from the present study. A total of 202 DLB patients were diagnosed according to the consensus criteria [1], with 172 patients diagnosed as probable DLB and 30 as possible DLB. A HMPAO SPECT scan was performed in 162 DLB patients, and occipital hypoperfusion was confirmed in 159 patients. Parkinsonism was assessed using the motor sign scores derived from the Unified Parkinson's Disease Rating Scale [8]. The following 11 items were rated on a scale from 0 to 4 : speech, tremor at rest (in any limb), facial expression, neck rigidity, right arm rigidity, left arm rigidity, right leg rigidity, left leg rigidity, posture, gait, and body bradykinesia. Total motor sign scores (range: 0-44) were used as an index of the severity of parkinsonism. Two patients had been treated with L-DOPA, 5 with donepezil, and 1 patient with risperidone prior to their first visit to our hospital. All patients who developed dementia 12 months or more after the onset of Parkinson disease were excluded. Disease duration was determined as the age at onset of dementia symptoms, provided by the caregiver, until present. 
Nagahama et al.: Effect of Aging on Cognition in DLB Patients

A total of $236 \mathrm{AD}$ patients were selected retrospectively as reference group to match age, education, and MMSE score with the DLB group. AD was diagnosed based on the DSM IV TR [9] and NINCDS/ADRDA criteria for probable AD [10]. Disease duration and the motor sign score were not evaluated in the AD patients. The number of subjects aged $<65$ years was small (11 in the DLB and 3 in the AD group), so they were excluded from the study. The study protocol has been approved by the Ethics Committee of Shiga Medical Center.

\section{Neuropsychological Examinations}

The following neuropsychological tests were routinely administered to all subjects by two experienced psychologists (T.O. and N.S.): the MMSE, cued recall, clock drawing test (CDT), copying a cube, verbal fluency (semantic fluency with names of fruits, and phonemic fluency with words beginning with "ka" in Japanese), Trail Making Test Part A (TM-A), and the Kohs Block Design Test. Cued recall was evaluated immediately following the delayed recall of 3 words in the MMSE, using the initial sound of each word as a cue. The CDT was scored according to the modified Shulman method (range: $0-5$ points) [11-13]. Copying a cube was quantitatively scored according to the criteria by Strub and Black [14] (range: 0-3 points). The results of the neuropsychological assessments were not part of the diagnostic process of DLB and AD.

\section{Statistical Analyses}

Our subjects were divided into three groups by age (65-74, 75-84, and 85-95 years old). To evaluate the effect of age on neuropsychological performance, we used a two-way analysis of variance (ANOVA), with diagnosis (DLB vs. AD) and age as the fixed factors. The main and interaction effects were tested at a critical level of $\alpha=0.05$. When an interaction effect was significant, we then examined the performance difference of the DLB and AD patients within each age group using one-way ANOVA with Bonferroni correction $(p<0.05 / 3)$.

\section{Results}

Table 1 shows the demographics and the neuropsychological data of the subjects. Two-way ANOVA showed a significant main effect of diagnosis on delayed recall $(\mathrm{F}=37.06$, $p<0.0001)$, cued recall $(\mathrm{F}=26.63, p<0.0001)$, CDT $(\mathrm{F}=29.28, p<0.0001)$, copying a cube $(\mathrm{F}=46.87, p<0.0001)$, category fluency $(\mathrm{F}=6.14, p=0.0136)$, phonemic fluency $(\mathrm{F}=18.20$, $p<0.0001)$, TM-A ( $\mathrm{F}=86.42, p<0.0001)$, and Kohs Block Design Test $(\mathrm{F}=52.62, p<0.0001)$. Patients with DLB showed a significantly better performance on the delayed and cued recalls than those with AD. On the other hand, DLB patients showed significantly poorer performance on CDT, copying a cube, category fluency, phonemic fluency, TM-A, and Kohs Block Design Test than AD patients. Two-way ANOVA also showed a significant main effect of age on education $(\mathrm{F}=4.93, p=0.0077)$, delayed recall $(\mathrm{F}=6.59, p=0.0015)$, cued recall $(\mathrm{F}=11.13$, $p<0.0001)$, CDT $(\mathrm{F}=6.16, p=0.0023)$, TM-A ( $\mathrm{F}=4.50, p=0.0116)$, category fluency $(\mathrm{F}=$ $11.98, p<0.0001)$, and phonemic fluency ( $\mathrm{F}=3.51, p=0.0307)$. Performances in these tests deteriorated with age, and the education level was lower in the older population, consistent with the general Japanese population. The MMSE score did not differ according to diagnosis or age groups. Disease duration $(\mathrm{F}=0.35, p=0.71)$ and the motor sign score $(\mathrm{F}=0.38, p=$ 0.68 ) did not differ among the age groups within the DLB patients.

There was a significant diagnosis-by-age interaction on delayed recall $(\mathrm{F}=3.84, p=$ $0.0223)$ and cued recall $(F=3.14, p=0.044)$ performances (Fig. 1). Post-hoc analyses showed significantly better performances in delayed and cued recalls in DLB patients than in agematched $\mathrm{AD}$ patients in the age groups $65-74$ years $(\mathrm{F}=20.80, p<0.0001$, and $\mathrm{F}=22.10$, 
Table 1. Demographics and neuropsychological data of the subjects

\begin{tabular}{|c|c|c|c|}
\hline & \multicolumn{3}{|l|}{ Age groups } \\
\hline & $65-74$ years & 75-84 years & $85-95$ years \\
\hline \multicolumn{4}{|l|}{ DLB group } \\
\hline \multicolumn{4}{|l|}{ Demographics } \\
\hline Patients, $n$ & 41 & 123 & 38 \\
\hline Age, years & $71.7 \pm 2.6$ & $79.3 \pm 2.9$ & $87.3 \pm 2.1$ \\
\hline Education, years & $9.9 \pm 2.7$ & $9.6 \pm 2.6$ & $8.7 \pm 2.2$ \\
\hline Disease duration, years & $1.7 \pm 1.9$ & $1.9 \pm 2.2$ & $2.0 \pm 1.9$ \\
\hline \multicolumn{4}{|l|}{ Neuropsychological assessment } \\
\hline Motor sign score & $5.6 \pm 5.1$ & $6.1 \pm 5.4$ & $5.2 \pm 5.3$ \\
\hline MMSE score & $21.8 \pm 3.9$ & $20.4 \pm 3.6$ & $20.3 \pm 4.0$ \\
\hline Delayed recall & $0.78 \pm 0.94$ & $0.43 \pm 0.72$ & $0.30 \pm 0.46$ \\
\hline Cued recall & $1.49 \pm 0.78$ & $0.94 \pm 0.86$ & $0.73 \pm 0.73$ \\
\hline Category fluency, /min & $7.5 \pm 3.3$ & $6.7 \pm 2.4$ & $5.7 \pm 2.2$ \\
\hline Phonemic fluency, /min & $5.5 \pm 2.9$ & $4.8 \pm 2.8$ & $4.1 \pm 2.6$ \\
\hline Clock drawing test & $3.80 \pm 1.26$ & $3.44 \pm 1.22$ & $3.13 \pm 1.28$ \\
\hline Trail Making Test Part A, s & $160.2 \pm 93.4$ & $179.6 \pm 100.8$ & $190.2 \pm 131.9$ \\
\hline Copying a cube & $1.54 \pm 1.14$ & $1.57 \pm 1.09$ & $1.66 \pm 1.05$ \\
\hline Kohs Block Design Test (IQ) & $52.2 \pm 27.6$ & $46.8 \pm 23.9$ & $48.7 \pm 21.9$ \\
\hline \multicolumn{4}{|l|}{ AD group } \\
\hline \multicolumn{4}{|l|}{ Demographics } \\
\hline Patients, $n$ & 67 & 121 & 48 \\
\hline Age, years & $70.5 \pm 2.9$ & $79.3 \pm 2.7$ & $87.5 \pm 1.9$ \\
\hline Education, years & $10.4 \pm 1.8$ & $9.6 \pm 2.3$ & $9.2 \pm 1.9$ \\
\hline \multicolumn{4}{|l|}{ Neuropsychological assessment } \\
\hline MMSE & $20.3 \pm 2.7$ & $20.1 \pm 2.8$ & $19.9 \pm 3.3$ \\
\hline Delayed recall & $0.18 \pm 0.42$ & $0.09 \pm 0.29$ & $0.15 \pm 0.41$ \\
\hline Cued recall & $0.76 \pm 0.78$ & $0.60 \pm 0.71$ & $0.52 \pm 0.65$ \\
\hline Category fluency, / min & $8.2 \pm 2.4$ & $7.2 \pm 2.4$ & $6.5 \pm 2.0$ \\
\hline Phonemic fluency, /min & $6.7 \pm 3.4$ & $5.8 \pm 3.0$ & $6.0 \pm 3.1$ \\
\hline Clock drawing test & $4.28 \pm 1.00$ & $4.28 \pm 0.99$ & $3.79 \pm 1.24$ \\
\hline Trail Making Test Part A, s & $78.7 \pm 37.9$ & $94.1 \pm 40.9$ & $119.2 \pm 54.9$ \\
\hline Copying a cube & $2.34 \pm 0.90$ & $2.22 \pm 0.82$ & $2.31 \pm 0.80$ \\
\hline Kohs Block Design Test (IQ) & $66.0 \pm 16.2$ & $63.9 \pm 14.3$ & $63.2 \pm 10.4$ \\
\hline
\end{tabular}

Data are shown as means \pm standard deviations. DLB, dementia with Lewy bodies; AD, Alzheimer disease.

$p<0.0001$, respectively) and 75-84 years $(\mathrm{F}=24.00, p<0.0001$, and $\mathrm{F}=11.27, p=0.0009)$; however, this difference was not observed in the age group 85-95 years $(\mathrm{F}=2.33, p=0.13$, and $\mathrm{F}=2.11, p=0.15$ ). There were no significant interactions between diagnosis and age in any of the other neuropsychological tests, suggesting that the effect of age was similar in DLB and $\mathrm{AD}$ patients for performances of tests other than the delayed and cued recalls.

\section{Discussion}

The present study demonstrated that performance of recent memory in DLB patients was significantly better than that of $A D$ patients in the age groups 65-74 and 75-84 years; however, memory impairment in DLB patients became more severe with advanced age, and was comparable with that of $\mathrm{AD}$ patients in the age group 85-95 years. In contrast to recent 


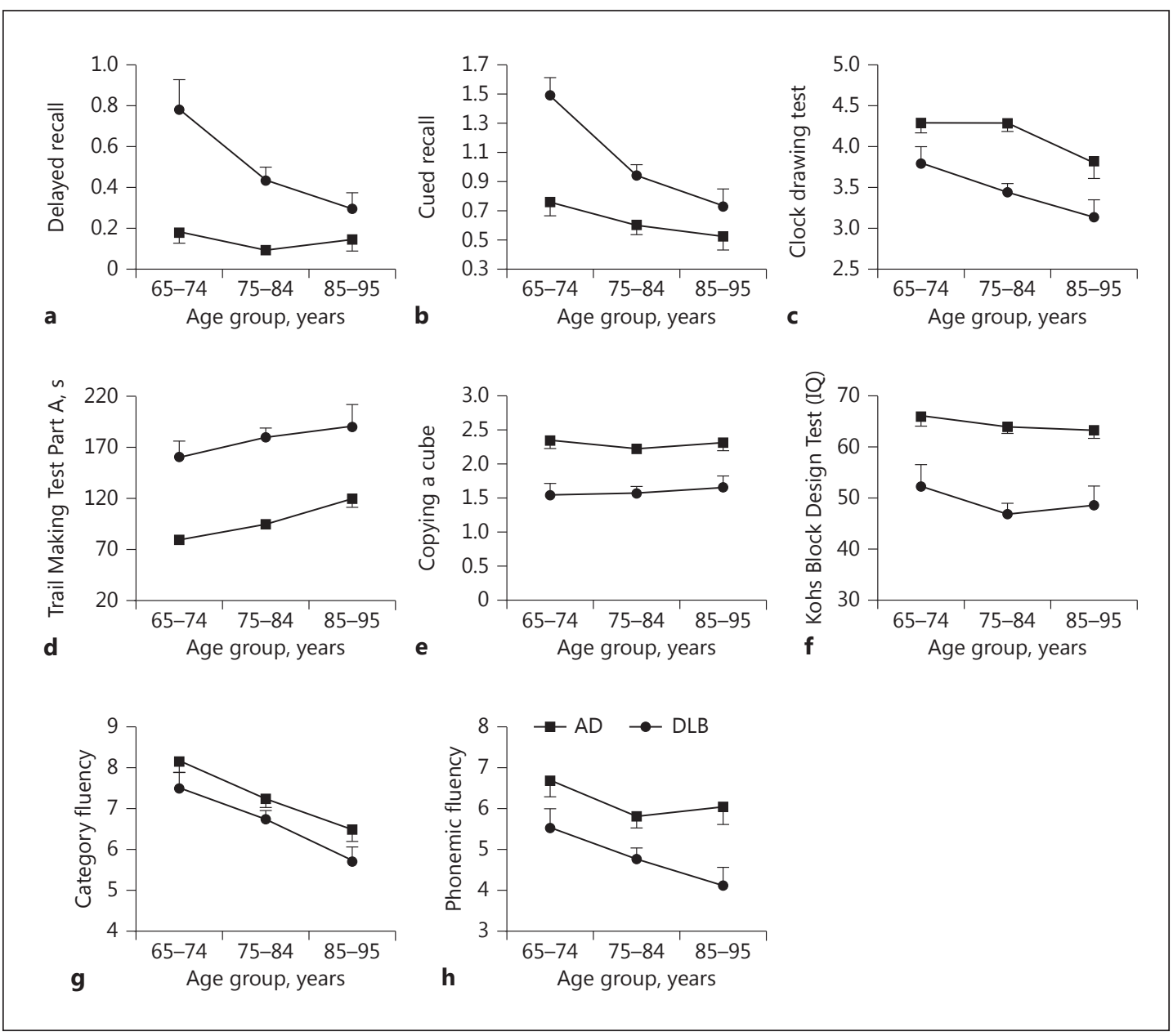

Fig. 1. Neuropsychological performances of the different age groups in patients with DLB and AD. Error bars indicate standard error of mean. DLB, dementia with Lewy bodies; AD, Alzheimer disease.

memory, there was no significant change in differences in the other neuropsychological performances between DLB and AD patients, such as visuospatial and executive functions, with advancing age.

Many previous studies have demonstrated that DLB patients show a relative preservation of memory function and greater impairment of attentional, executive, and visuospatial functions compared with AD patients [2-4]. Clinically, this "double dissociation" can help differentiate DLB from AD [1]. Overall, the results of the present study were consistent with previous studies [2-4]. Patients with DLB showed significantly better performance on delayed and cued recalls than AD patients, and significantly poorer performance on executive (category fluency, phonemic fluency, CDT, and TM-A) and visuo-constructional tasks (CDT, copying a cube, and Kohs Block Design Test). In addition, age influenced several executive task performances (category fluency, phonemic fluency, TM-A, and CDT) in both DLB and AD patients to the same degree. These results may reflect the deterioration of executive function with advancing age [15].

The present study demonstrated that the nature of memory impairment in DLB patients changed with age. DLB patients in the young-old and old-old age groups showed significantly better memory performances when compared with AD patients, whereas memory perfor- 
mances of DLB patients in the oldest-old age group were similar to those of AD patients. This difference is likely not due to dementia severity, because disease duration, MMSE score, and motor impairment were comparable among the different age groups of DLB.

We speculate that pronounced memory impairment at an older age of DLB onset may be associated with the increase of coexisting AD pathology. Positron emission tomography studies have shown that amyloid- $\beta$ positivity increases with aging in DLB patients, suggesting the coexistence of $A D$ pathology with advancing age [16]. A pathological study reported that with advancing age the prevalence of mixed DLB/AD pathology slightly increased, and that of pure DLB pathology decreased, suggesting an increased proportion of mixed DLB/AD pathology in older DLB patients [17]. Medial temporal lobe atrophy (MTA) is a well-recognized feature of $\mathrm{AD}$, and has been associated with memory impairment in $\mathrm{AD}[18,19]$. An autopsy-confirmed study revealed that MTA on MRI was strongly associated with a high Braak stage and neurofibrillary tangle density, and that it was a highly accurate diagnostic marker for AD pathology [20]. Both pathological and neuroimaging studies have demonstrated that DLB patients with coexisting AD pathology or amyloid- $\beta$ positivity showed very similar patterns of cortical atrophy, including in the hippocampal/parahippocampal area, to those of $A D$ patients, whereas DLB patients without AD pathology or amyloid- $\beta$-negative DLB patients showed lower MTA [21, 22]. MRI studies have shown that MTA in DLB was associated with memory impairment [23, 24]. A high-magnetic field MRI study revealed that the cornu ammonis 1 within the hippocampus, which is especially vulnerable to AD pathology, was relatively preserved in DLB compared with AD, and cornu ammonis 1 volume was observed to be correlated with recent memory and delayed recall scores in DLB [24]. Thus, the pronounced memory disturbance observed in DLB patients of advanced age may be, at least in part, due to the increased rate of coexisting AD pathology.

Several limitations should be noted in the present study. First, all dementia subjects were diagnosed clinically, and were not proven pathologically. Second, because the AD patients were retrospectively selected, disease duration and motor sign score were not estimated in the AD group. Third, MTA was not evaluated in the present study because many subjects underwent CT imaging but not MRI. Fourth, biomarkers such as amyloid- $\beta$ or tau in the cerebrospinal fluid would strengthen the result of the study, but we did not estimate such biomarkers. At last, memory performances were assessed only with 3-word delayed recall tests in this study. A neuropsychological assessment with more detailed memory tests may give further information. The relationships between age, memory impairment, pathological changes, biomarkers, and MTA in DLB patients should be further examined in future investigations.

\section{Conclusion}

In the present study, we demonstrated that memory impairment in DLB patients became more pronounced with older age, and that it was comparable to that of AD patients in the oldest-old age group. The neuropsychological "double dissociations" (i.e., the relative preservation of memory and greater visuospatial/executive dysfunction) are clues to differentiate DLB from $\mathrm{AD}$, but this is not the case in oldest-old patients, because memory performance in DLB may be indistinguishable from that in $\mathrm{AD}$ in the oldest-old age group.

\section{Disclosure Statement}

The authors have no conflicts of interest to report. 
Nagahama et al.: Effect of Aging on Cognition in DLB Patients

\section{References}

1 McKeith IG, Dickson DW, Lowe J, et al: Diagnosis and management of dementia with Lewy bodies: third report of the DLB Consortium. Neurology 2005;65:1863-1872.

-2 Collerton D, Burn D, McKeith I, O’Brien J: Systematic review and meta-analysis show that dementia with Lewy bodies is a visual-perceptual and attentional-executive dementia. Dement Geriatr Cogn Disord 2003;16:229237.

-3 Oda H, Yamamoto Y, Maeda K: Neuropsychological profile of dementia with Lewy bodies. Psychogeriatrics 2009;9:85-90.

4 Metzler-Baddeley C: A review of cognitive impairments in dementia with Lewy bodies relative to Alzheimer's disease and Parkinson's disease with dementia. Cortex 2007;43:583-600.

5 Kosaka K: Diffuse Lewy body disease in Japan. J Neurol 1990;237:197-204.

6 6 Merdes AR, Hansen LA, Jeste DV, Galasko D, Hofstetter CR, Ho GJ, Thal LJ, Corey-Bloom J: Influence of Alzheimer pathology on clinical diagnostic accuracy in dementia with Lewy bodies. Neurology 2003;60:1586-1590.

7 Kaiser NC, Melrose RJ, Liu C, Sultzer DL, Jimenez E, Su M, Monserratt L, Mendez MF: Neuropsychological and neuroimaging markers in early versus late-onset Alzheimer's disease. Am J Alzheimers Dis Other Demen 2012;27:520-529.

-8 Scarmeas N, Hadjigeorgiou GM, Papadimitriou A, Dubois B, Sarazin M, Brandt J, Albert M, Marder K, Bell K, Honig LS, Wegesin D, Stern Y: Motor signs during the course of Alzheimer disease. Neurology 2004;63:975982.

9 American Psychiatric Association: Diagnostic and Statistical Manual of Mental Disorders, ed 4, revised. Washington, APA, 2000.

10 McKhann G, Drachman D, Folstein M, Katzman R, Price D, Stadlan EM: Clinical diagnosis of Alzheimer's disease: report of the NINCDS-ADRDA Work Group under the auspices of Department of Health and Human Services Task Force on Alzheimer's Disease. Neurology 1984;34:939-944.

-11 Nagahama Y, Okina T, Suzuki N, Nabatame H, Matsuda M: Neural correlates of impaired performance on the clock drawing test in Alzheimer's disease. Dement Geriatr Cogn Disord 2005;19:390-396.

12 Nagahama Y, Okina T, Suzuki N, Matsuda M: Cerebral substrates related to impaired performance in the clockdrawing test in dementia with Lewy bodies. Dement Geriatr Cogn Disord 2008;25:524-530.

13 Shulman KI: Clock-drawing: is it the ideal cognitive screening test? Int J Geriatr Psychiatry 2000;15:548-561.

14 Strub RL, Black FW: The Mental Status Examination in Neurology, ed 4. Philadelphia, FA Davis, 2000.

15 Harada CN, Natelson Love MC, Triebel KL: Normal cognitive aging. Clin Geriatr Med 2013;29:737-752.

-16 Ossenkoppele R, Jansen WJ, Rabinovici GD, et al: Prevalence of amyloid PET positivity in dementia syndromes: a meta-analysis. JAMA 2015;313:1939-1949.

$\checkmark 17$ Jellinger KA, Attems J: Prevalence and pathology of dementia with Lewy bodies in the oldest old: a comparison with other dementing disorders. Dement Geriatr Cogn Disord 2011;31:309-316.

-18 Mori E, Yoneda Y, Yamashita H, Hirono N, Ikeda M, Yamadori A: Medial temporal structures relate to memory impairment in Alzheimer's disease: an MRI volumetric study. J Neurol Neurosurg Psychiatry 1997;63:214221.

-19 Smits LL, Tijms BM, Benedictus MR, Koedam EL, Koene T, Reuling IE, Barkhof F, Scheltens P, Pijnenburg YA, Wattjes MP, van der Flier WM: Regional atrophy is associated with impairment in distinct cognitive domains in Alzheimer's disease. Alzheimers Dement 2014;10:S299-S305.

20 Burton EJ, Barber R, Mukaetova-Ladinska EB, Robson J, Perry RH, Jaros E, Kalaria RN, O’Brien JT: Medial temporal lobe atrophy on MRI differentiates Alzheimer's disease from dementia with Lewy bodies and vascular cognitive impairment: a prospective study with pathological verification of diagnosis. Brain 2009; 132:195-203.

-21 Nedelska Z, Ferman TJ, Boeve BF, Przybelski SA, Lesnick TG, Murray ME, Gunter JL, Senjem ML, Vemuri P, Smith GE, Geda YE, Graff-Radford J, Knopman DS, Petersen RC, Parisi JE, Dickson DW, Jack CR Jr, Kantarci K: Pattern of brain atrophy rates in autopsy-confirmed dementia with Lewy bodies. Neurobiol Aging 2015;36: 452-461.

22 Shimada H, Shinotoh H, Hirano S, Miyoshi M, Sato K, Tanaka N, Ota T, Fukushi K, Irie T, Ito H, Higuchi M, Kuwabara S, Suhara T: Beta-amyloid in Lewy body disease is related to Alzheimer's disease-like atrophy. Mov Disord 2013;28:169-175.

-23 Barber R, McKeith IG, Ballard C, Gholkar A, O’Brien JT: A comparison of medial and lateral temporal lobe atrophy in dementia with Lewy bodies and Alzheimer's disease: magnetic resonance imaging volumetric study. Dement Geriatr Cogn Disord 2001;12:198-205.

24 Mak E, Li S, Williams GB, Watson R, Firbank M, Blamire A, O’Brien J: Differential atrophy of hippocampal subfields: a comparative study of dementia with Lewy bodies and Alzheimer disease. Am J Geriatr Psychiatry 2016;24:136-143. 